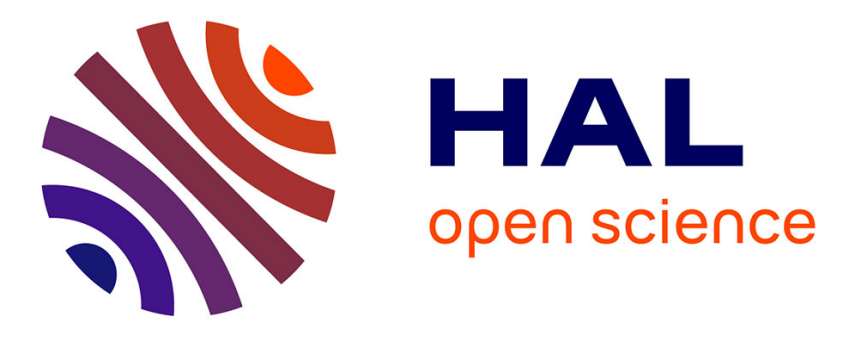

\title{
Impact of Avatar Anthropomorphism and Task Type on Social Presence in Immersive Collaborative Virtual Environments
}

Charlotte Dubosc, Geoffrey Gorisse, Olivier Christmann, Sylvain Fleury, Killian Poinsot, Simon Richir

\section{To cite this version:}

Charlotte Dubosc, Geoffrey Gorisse, Olivier Christmann, Sylvain Fleury, Killian Poinsot, et al.. Impact of Avatar Anthropomorphism and Task Type on Social Presence in Immersive Collaborative Virtual Environments. 2021 IEEE Conference on Virtual Reality and 3D User Interfaces Abstracts and Workshops (VRW), Mar 2021, Porto (virtual ), Portugal. pp.438-439, 10.1109/VRW52623.2021.00101 . hal-03371513

\section{HAL Id: hal-03371513 \\ https://hal.science/hal-03371513}

Submitted on 8 Oct 2021

HAL is a multi-disciplinary open access archive for the deposit and dissemination of scientific research documents, whether they are published or not. The documents may come from teaching and research institutions in France or abroad, or from public or private research centers.
L'archive ouverte pluridisciplinaire HAL, est destinée au dépôt et à la diffusion de documents scientifiques de niveau recherche, publiés ou non, émanant des établissements d'enseignement et de recherche français ou étrangers, des laboratoires publics ou privés. 


\title{
Impact of Avatar Anthropomorphism and Task Type on Social Presence in Immersive Collaborative Virtual Environments
}

\author{
Charlotte Dubosc* Geoffrey Gorisse \\ Olivier Christmann \\ Sylvain Fleury \\ Killian Poinsot \\ Simon Richir
}

Arts et Métiers Insitute of Technology, LAMPA, Changé France

\begin{abstract}
Eliciting a sense of social presence is necessary to create believable multi-user situations in immersive virtual environments. To be able to collaborate in virtual worlds, users are represented by avatars (virtual characters controlled in real time) allowing them to interact with each other. We report a study investigating the impact on social presence of both non-human avatars' facial properties and of the type of collaborative task being performed by the users (asymmetric collaboration versus negotiation). While we observed no significant impact of facial properties, both co-presence and perceived message understanding scores were significantly higher during the negotiation task.
\end{abstract}

Index Terms: Human-centered computing-Virtual reality-;-

\section{INTRODUCTION AND RELATED WORK}

Current virtual reality (VR) technologies enable remote collaboration in immersive virtual environments through virtual characters. This study focuses on social presence which refers to the degree of initial awareness, allocated attention, the capacity for both content and affective comprehension, and the capacity for affective and behavioral interdependence during a mutual interaction with a perceived entity [3]. In the frame of our experiment, we focus especially on co-presence, defined as the sense of being there with others, and perceived message understanding, the ability to understand the message being received from the interactant as well as the perception of the interactant's level of message understanding, as they appeared to be particularly relevant considering the tasks of our experiment. The first line of this research consists of evaluating how avatars' facial properties can affect the way participants perceive each other and collaborate. We chose to resort to robotic avatars presenting non-anthropomorphic body schema (Fig. 1) to be in line with several VR applications currently available to the general public. The second goal is to compare two remote collaborative situations: an asymmetrical collaboration and a negotiation task.

The meta-analysis of $\mathrm{Oh}$ et al. [7] lists numerous studies and identifies several immersive, contextual and psychological factors impacting the sense of social presence in virtual environments. Among the different predictors identified, we will focus on the influence of visual and behavioral aspects of virtual characters as well as on the impact of the type of task that can affect social presence. Garau et al. [2] demonstrated that humanoid avatars with a high level of visual fidelity and realistic gaze induce a higher sense of social presence than avatars with a random gaze system. This experiment also highlights the fact that the higher the visual fidelity, the higher the requirements for realistic behavior. In terms of appearance, and especially in terms of anthropomorphism level, it has been demonstrated that users seem to be less prone to accept virtual characters' flaw as

*e-mail:charlotte.dubosc@ensam.eu they get closer to realistic human appearance [6]. Recently, previous experiments observed higher co-presence scores with partial avatars (floating head and hands), sometimes even higher than with full body characters presenting low tracking fidelity $[4,5]$. It appears that a partial virtual body could be better than a technically limited full body representation.

Very few previous studies investigated the task type impact on the sense of social presence [7]. However, communication is an important part of collaborative immersive virtual environments. Nonverbal communication can be divided in two categories : conscious non-verbal and unconscious non-verbal. The first one adds an emotional valence and can support the speaker's words when used in combination with verbal communication. Gestures and facial expressions appear to improve users' sense of co-presence in virtual environments [1].

\section{Materials and Methods}

To study how anthropomorphism can affect participants' sense of social presence in both the asymmetric collaborative task (AC) and the negotiation task $(\mathrm{N})$, we designed three avatars. Following a between-subject design, we recruited different participants in six distinct groups, one for each avatar and task. 36 participants (10 females ad 26 males) were recruited for the AC task and 36 participants (11 females and 25 males) were recruited for the $\mathrm{N}$ task. Each pair of participants embodies one of the three avatars classified according to their facial properties (Fig. 1):

- Robot 1 (R1): screen based face that displays both the eyes and the mouth animated using different set of textures. The robot's mouth is animated when the user is talking using a sequence of six textures.

- Robot 2 (R2): physical eyes and virtual mouth. The virtual mouth is an audio visualizer displaying bars matching the intensity of the user's vocal frequencies.

- Robot 3 (R3): physical eyes and jaw. The physical jaw moves according to the user's voice intensity.

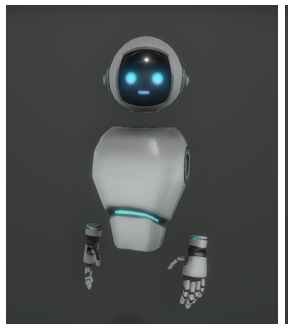

(a) R1

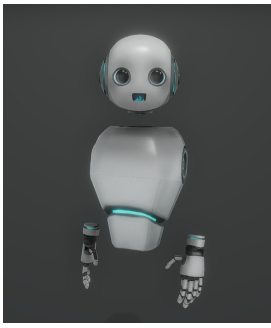

(b) R2

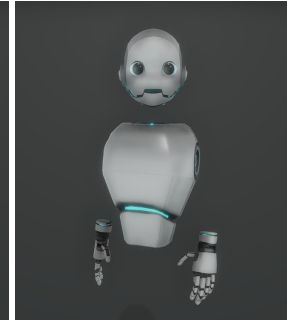

(c) R3
Figure 1: Avatars of the experiment 


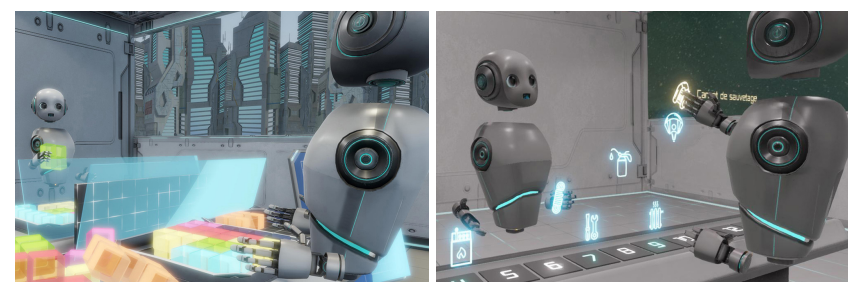

Figure 2: Participants performing the collaborative task (left) and the negotiation task (right).

We developed two tasks presenting potential remote collaborative situations (Fig. 2). The first task consists in an asymmetric collaboration. Participants have to solve two puzzle games. As a training phase, they complete the first puzzle independently following the plan and using the parts available on their own virtual table. When both participants have completed their first puzzle, they must collaborate to complete the second one explaining alternately where to place the $3 \mathrm{D}$ parts on the game boards, as each participant has the plan corresponding to their partner's puzzle. In this kind of situation, participants focus most of the time on the game board relying on verbal communication to follow their partner's instruction.

The second task presents a negotiation situation where participants face each other and must discuss to reach an agreement. We developed an adapted version of the NASA's survival on the moon exercise. Partners must collaborate to classify objects from 1 to 15 in order to survive after forced landing on the moon's surface. We designed an interface composed of 15 icons (one for each object) ensuring that participants will face each other during the whole experience. As soon as they agree, the task is over.

At the end of the experiment, the participants fill the social presence questionnaire composed of two dimensions: co-presence and perceived message understanding dimensions of the Networked Mind Social Presence Measure [3]. These data are then analyzed separately for each task. A cross-analysis was conduced afterward to analyze the effect of the task type on the sense of social presence.

\section{Results AND discussion}

Data were tested for normality and homogeneity of variance. Shapiro-Wilk Tests revealed that some variables were not normally distributed $(p<0.05)$. However, considering that Levene Tests showed that the variances were not significantly different, we used parametric tests to analyze the data. Results are considered significant when $p<0.05$. A two-way between-groups analysis of variance was conducted to explore the impact of anthropomorphism and of the type of task on the co-presence and the perceived message understanding dimensions of social presence. Participants were divided into six groups according to the robot they controlled and the type of task they carried out.

A two-way between-groups analysis of variance was conducted to explore the impact of anthropomorphism and of the type of task on the co-presence dimension of social presence. The interaction effect between anthropomorphism and task was not statistically significant, $F(2,66)=3.03, p=0.055$. There was a statistically significant main effect of task type, $F(1,66)=4.55, p=0.037$. The effect size was small (partial eta squared $=0.065$ ). The mean co-presence score for the negotiation task $(M=6.41, S D=0.72)$ was superior to the asymmetric collaboration task $(M=6, S D=0.90)$. The main effect for anthropomorphism, $F(2,66)=0.47, p=0.63$, did not reach statistical significance. Another two-way betweengroups analysis of variance was conducted to explore the effect of anthropomorphism and of the type of task on the perceived message understanding dimension of social presence. The interaction effect between anthropomorphism and task was not statistically significant, $F(2,66)=1.22, p=0.302$. There was a statistically significant main effect of task type, $F(1,66)=29.96, p<0.001$. The effect size was small (partial eta squared $=0.312$ ). The mean perceived message understanding score for the negotiation task $(M=6.32, S D=0.67)$ was superior to the asymmetric collaboration task $(M=5.34, S D=$ $0.84)$. The main effect for anthropomorphism, $F(2,66)=0.66, p=$ 0.519 , did not reach statistical significance.

The analysis revealed no significant differences concerning the impact of anthropomorphism on participants' sense of social presence. Previous research demonstrated that users can feel a high sense of co-presence using partial avatars (floating head and hands) [4]. However, we expected some differences with a potential improvement as the facial properties of the avatars get closer to human appearance. We argue that it could be linked to a potential ceiling effect. Indeed, participants reported a very high sense of co-presence and a clear understanding of their partner messages with every avatar conditions. These results could be explained by the fact that the effect of facial properties can be minor compared to the impact of verbal and non-verbal communication (body language, hand movements, etc.). As observed in previous experiments [6], it is also possible that users are more sensitive to realistic facial properties of virtual humans and that such differences do not matter so much on robotic characters. Nevertheless, our results seem to indicate that it is possible to induce a high sense of social presence in immersive virtual environments using non-human virtual characters.

Our results demonstrate that social presence is context-sensitive. In terms of co-presence, a significant difference between the two tasks was observed in favor of the negotiation task. Participants experienced a higher sense of co-presence with the first robot when they had to reach an agreement. We also observed several significant differences concerning the perceived message understanding dimension. It appeared that the participants were more able to understand their partners in the negotiation task. An explanation could be that visual contact between the participants favored non-verbal communication, especially during the negotiation task which was designed to ensure that the participants face each other for most of the immersion period. Both verbal and non-verbal communication could have led to a flawless interaction between the participants, which would have improved their overall sense of social presence.

\section{REFERENCES}

[1] J. Casanueva and E. Blake. The Effects of Avatars on Co-presence in a Collaborative Virtual Environment. In Hardware, Software and Peopleware: Proceedings of the Annual Conference of the South African Institute of Computer Scientists and Information Technologists, 2001.

[2] M. Garau, M. Slater, V. Vinayagamoorthy, A. Brogni, A. Steed, and M. A. Sasse. The Impact of Avatar Realism and Eye Gaze Control on Perceived Quality of Communication in a Shared Immersive Virtual Environment. In Proceedings of the SIGCHI Conference on Human Factors in Computing Systems, CHI '03, pages 529-536, New York, NY, USA, 2003. ACM.

[3] C. Harms and F. Biocca. Internal consistency and reliability of the networked minds measure of social presence. In M. Alcaniz and B. Rey, editors, Proceedings of the 2004 Seventh Annual International Workshop: Presence, 2004.

[4] P. Heidicker, E. Langbehn, and F. Steinicke. Influence of avatar appearance on presence in social vr. In 2017 IEEE Symposium on 3D User Interfaces (3DUI), pages 233-234, 2017.

[5] F. Herrera, S. Y. Oh, and J. N. Bailenson. Effect of behavioral realism on social interactions inside collaborative virtual environments. PRESENCE: Virtual and Augmented Reality, 27(2):163-182, 2020.

[6] R. McDonnell, M. Breidt, and H. H. Bülthoff. Render me real?: Investigating the effect of render style on the perception of animated virtual humans. ACM Trans. Graph., 31(4):91:1-91:11, July 2012.

[7] C. S. Oh, J. N. Bailenson, and G. F. Welch. A systematic review of social presence: Definition, antecedents, and implications. Frontiers in Robotics and AI, 5:114, 2018. 